\title{
RUPTURED BRONCHIAL ARTERY ANEURYSM: CASE REPORT
}

\author{
Filip Čečka1, Jan Raupach ${ }^{2}$, Petr Hoffmann², Petr Motyčka', Milan Štětina ${ }^{3}$, Čestmír Neoral ${ }^{4}$
}

Charles University in Prague, Faculty of Medicine and University Hospital Hradec Králové, Czech Republic: Department of Surgery ${ }^{1}$, Department of Radiology ${ }^{2}$, Department of Cardiosurgery ${ }^{3}$; Palacký University, Faculty of Medicine and University Hospital Olomouc, Czech Republic: Department of Surgery ${ }^{4}$

Summary: Bronchial artery aneurysm is a very rare condition. Its rupture can cause a life threatening hemorrhage. We report a case of a 50-year-old patient with a ruptured bronchial artery aneurysm who presented with epigastric pain. It is the first published case with such signs of a rupture of a bronchial artery aneurysm. CT scan confirmed the diagnosis. The patient was treated with endovascular embolization.

Key words: Bronchial artery aneurysm; Endovascular embolization; Epigastric pain

\section{Introduction}

Bronchial artery aneurysm is a very rare condition. The aneurysms are usually asymptomatic until the point of rupture. The most frequent signs of rupture are hemoptysis or hematemesis and dysphagia. We present a case of a patient whose first sign of bronchial artery aneurysm rupture was epigastric pain.

\section{Case Report}

A 50-year-old male came to the acute admission unit for sharp stabbing epigastric pain which had lasted for four days. The patient reported severe sharp pain reaching behind his sternum. He had been treated for arterial hypertension for about one year with the combination of Bisoprolol (Bisoprolol, Merckle GmbH, Germany) and Rhefluin (Amilorid and Hydrochlorothiazid, Zentiva a.s., Czech Republic) and he had abandoned the treatment about three months before admission.

The patient also reported that he had eaten a meal with a plastic fork about ten days before the admission and one fork tooth had been missing after the meal. Cardiac etiology, foreign body (tooth of the plastic fork) in the gastrointestinal tract, duodenal ulcer perforation, thoracic aorta dissection were considered as the cause of the pain.

Gastroscopy excluded foreign body in the gastrointestinal tract and duodenal ulcer. CT scan revealed the diagnosis: a hypertrophic bronchial artery with aneurysm was visible, with large mediastinal hematoma and hematoma in the right pleural cavity (Fig. 1).

The final diagnosis was an aneurysm of the right bronchial artery with rupture and bleeding. Angiography and embolization attempt was considered as the treatment of choice.

During the intervention we inserted a RC 1 catether into the hypertrophic winding right bronchial artery, where the aneurysm was, with a diameter of about $10 \mathrm{~mm}$. The se-

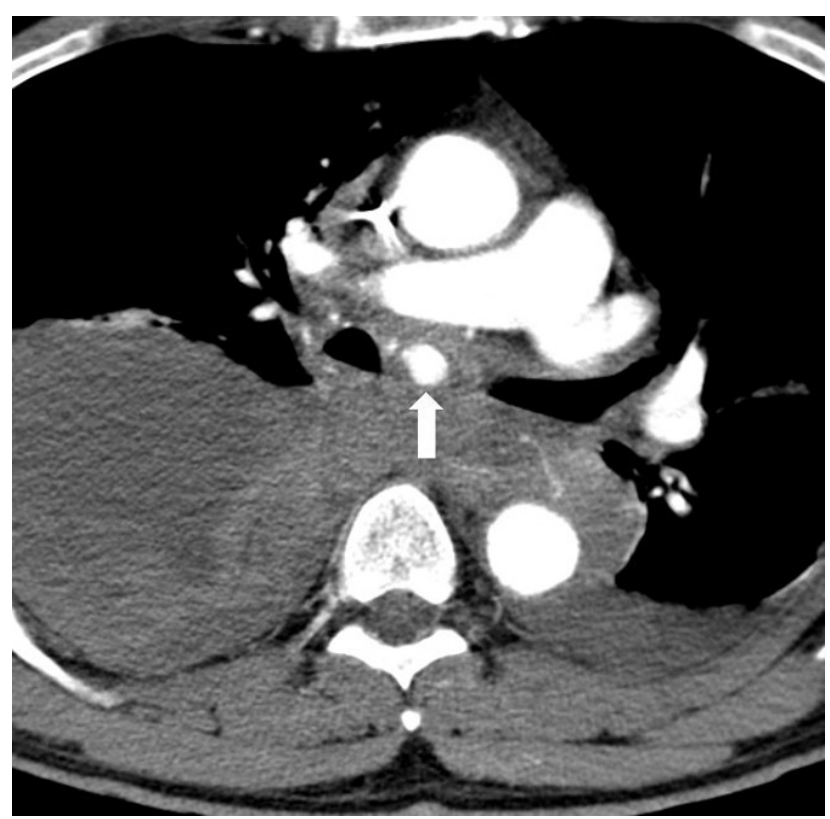

Fig. 1: Enhanced CT scan focused at the trunk and branches of the pulmonary artery and the thoracic aorta. The hypertrophic bronchial artery with the aneurysm, under the carina (arrow) is arising from the thoracic aorta. This aneurysm is the source of bleeding into the mediastinum and both pleural cavities, more into the right side. 

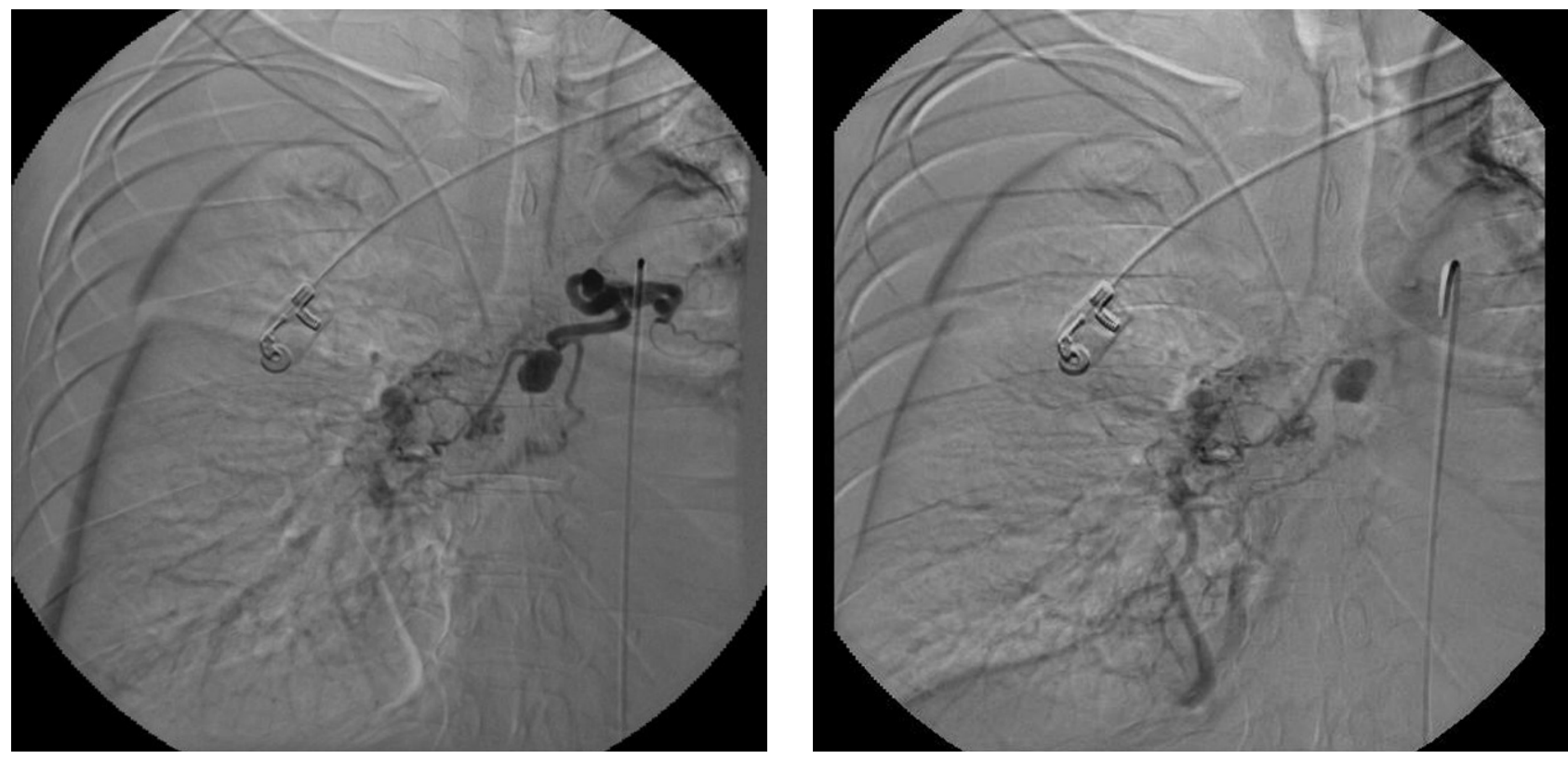

Fig. 2 and 3: Selective angiography shows the hypertrophic bronchial artery with the aneurysm. The bronchial artery aneurysm formed a shunt with part of the pulmonary artery.

lective embolization of the aneurysm was not successful due to the winding shape of the afferent artery, and therefore we performed proximal embolization of the bronchial artery only a short distance behind the aorta. We used six $3 \mathrm{~mm}$ Vortex coils (Boston Scientific, USA). The procedure took 160 minutes and was without complications (Figs. 2, 3). The patient was discharged on the eighth day after the procedure. At the time of this report, 24 months after the event, the patient has no further complaint regarding the aneurysm.

\section{Discussion}

Seventy-two cases of bronchial artery aneurysm rupture have been published in the world literature so far (1). A brief review of management of bronchial artery aneurysm was recently published by Yajima et al. (8). The cause of the aneurysm could be brochiectasis, recurrent bronchopulmonary inflammation, mycotic origin, trauma (9) or systemic vascular disease, e.g. Rendu-Osler-Weber syndrome or ateriosclerosis $(2,6)$.

The bronchial artery aneurysms are usually asymptomatic until they rupture. The rupture can be a life-threatening condition. In most cases it presents with hemoptysis because the aneurysm ruptures into the lung parenchyma or a bronchus. If the aneurysm ruptures into the mediastinum, it will form a hematoma which will obstruct the oesophagus, or eventually rupture into the oesophagus, and then present with dysphagia or hematemesis (7). In some cases the bronchial artery aneurysm rupture can have a clinical picture indistinguishable from thoracic aorta dissection (2). A case of bronchial artery aneurysm disguised as esophageal leiomyoma was reported (7).
The diagnosis is usually based on the imaging methods. Ultrasound scans can reveal hemothorax. CT scans or MRI have greater benefit, displaying hypertrophic artery with the aneurysm, and the diagnosis is confirmed by the angiography (3).

Endovascular embolization is the method of choice. The embolization can be achieved with metal microcoils. It is necessary to insert the microcatether into the aneurysm. The goal is to homogenously fill the aneurysm and to exclude it from blood flow. If possible, it appears optimal to commence the embolization in the vessel behind the aneurysm, occlude the aneurysm sac and continue with the embolization of afferent vessel, so called trapping technique. This technique prevents retrograde filling of the aneurysm. Another endovascular technique is embolization with N-butyl-2-cyanoacrylate (NBCA; Histoacryl, B. Braun, Germany). The microcatether is inserted into the feeding vessel and the NBCA can be diluted by iodized oil Lipidol (Lipidol UltraFuide, Guebert, France). It is possible to embolize the feeding vessel, the aneurysm and the efferent vessel. This technique is used in tortuous elongated vessels where it is not possible to insert the catheter inside the aneurysm $(1,4)$.

Only in cases where the endovascular technique fails, or it is not possible to perform it (due to hemodynamic instability of the patient, iodine contrast allergy, etc.), surgical procedure should be performed $(3,4,6)$. Surgical aneurysmectomy is also necessary in case of unsuccessful embolization or in case of continuous bleeding (9).

Management of an asymptomatic aneurysm remains controversial. There are no known predictors of rupture of the aneurysm, and the rupture can be a life threatening condition. Therefore asymptomatic aneurysms should be treated 
as well. Some authors recommend preventive thoracotomy with an aneurysmectomy, or even lobectomy (5). However, we are convinced that miniinvasive endovascular treatment is the method of choice.

In our patient, the epigastric pain was caused by hematoma in mediastinum. According to our best knowledge, this is the first published case of a patient with bronchial artery aneurysm presenting with epigastric pain. Treating the aneurysm, we prefered endovascular intervention, which proved to be optimal in this case.

\section{References}

1. Aburano H, Kawamori $\mathrm{Y}$, Horiti $\mathrm{Y}$, et al. Bronchial artery aneyrysm embolization with NBCA. Cardiovasc Intervent Radiol 2006;29:1141-3.
2. Hall RJ, Miller GA, Kerr IH. Ruptured bronchial artery aneurysm mimicking aortic dissection. Br Heart J 1977;39:909-10.

3. Karmy-Jones R, Hastreiter D, Burdick T. Hemothorax complicating bronchial artery aneurysm. Can Respir J 2005;12:279-81.

4. Pugnale M, Portier F, Lamarre A, et al. Hemomediastinum caused by rupture of a bronchial artery aneurysm: successful treatment by embolization with N-butyl2-cyanoacrylate. J Vasc Interv Radiol 2001;12:1351-2.

5. Saito Y, Ueda Y, Imamura H, Okamura A. Operative aneurysmectomy and middle lobectomy for asymptomatic bronchial artery aneurysm in young patient. Eur $\mathbf{J}$ Cardiothorac Surg 2000;19:366-9.

6. Sakuma K, Takase K, Saito H, Zuguchi M, Tabayashi K. Bronchial artery aneurysm treated with percutaneous transluminal coil embolization. Jpn J Thorac Cardiovasc Surg 2001;49:330-2.

7. Tringali S, Tiffet $\mathrm{O}$, Berger JL, Cuilleret J. Bronchial artery aneurysm disguised as a leiomyoma of the esophagus. Ann Thorac Surg 2002;73:632-3.

8. Yajima N, Tsutsui H, Yoshioka T, et al. Gigantic bronchial artery aneurysm treated with transcatheter arterial embolization: a case report. Angiology 2009;59: 757-60.

9. Yanagihara K, Ueno Y, Kobayashi T, Isobe J, Itoh M. Bronchial artery aneurysm. Ann Thorac Surg 1999;67:854-5.

Submitted December 2008. Accepted December 2008.

Corresponding author:

Filip Čečka, M.D., Charles University in Prague, Faculty of Medicine and University Hospital Hradec Králové, Sokolská 581, 50005 Hradec Králové, Czech Republic; e-mail: filip.cecka@seznam.cz 